\title{
Nuclear modification of strange and light-flavour hadrons measured with ALICE at the LHC
}

\section{Domenico ELIA ${ }^{* \dagger}$}

INFN Sezione di Bari, Bari (Italy)

E-mail: Domenico.Elia@ba.infn.it

\begin{abstract}
The ALICE experiment at CERN was designed to study the properties of the strongly-interacting hot and dense matter created in heavy-ion collisions at the LHC energies. Hard partons propagating through such a medium lose energy via multiple scattering and gluon radiation: this reflects in a modification of the transverse momentum $\left(p_{\mathrm{T}}\right)$ spectra of the final-state hadrons since their yields at high $p_{\mathrm{T}}$ are suppressed compared to the reference values from a simple superposition of incoherent proton-proton collisions.

Results on the suppression patterns for identified hadrons with and without strangeness content can be connected with the predicted energy loss dependence on the parton mass, while the comparison of baryon and meson suppressions at high $p_{\mathrm{T}}$ can probe different energy losses for quarks and gluons. In addition, results from $\mathrm{Pb}-\mathrm{Pb}$ collisions compared with the corresponding measurements carried out in $\mathrm{p}-\mathrm{Pb}$ allow to investigate possible contributions due to initial-state nuclear matter effects.
\end{abstract}

38th International Conference on High Energy Physics

3-10 August 2016

Chicago, USA

* Speaker.

${ }^{\dagger}$ On behalf of the ALICE Collaboration. 


\section{Introduction}

Hard partons propagating through the hot and dense strongly-interacting matter created in ultra-relativistic heavy-ion collisions (Quark Gluon Plasma, QGP [1]) are predicted to lose energy via multiple scattering and gluon radiation. As a result, the yield of final-state hadrons at high transverse momentum will be suppressed compared to the reference value from a simple superposition of incoherent proton-proton collisions $[2,3]$. This modification of the $p_{\mathrm{T}}$ spectra, quantified by the nuclear modification factor $\left(R_{\mathrm{AA}}\right)$ and measured for light-flavour hadrons with different quark content, allows to study the parton energy-loss mechanisms and medium properties. Similar measurements performed in $\mathrm{p}-\mathrm{A}$ collisions $\left(R_{\mathrm{pA}}\right)$ may help in determining whether initial or final state nuclear effects play a role in the suppression of hadron production observed in A-A.

The design of the ALICE experiment was optimized to study the properties and the evolution of the hot and dense matter created in heavy-ion collisions at the LHC. Its apparatus consists of a central barrel with a large solenoid providing a $0.5 \mathrm{~T}$ magnetic field for tracking and particle identification, plus a dimuon spectrometer equipped with its own dipole magnet with a $0.7 \mathrm{Tm}$ field integral, and other forward detectors for triggering and centrality selection. It has specific unique capabilities for measuring the production of strange and light-flavour hadrons over a wide range of transverse momentum, from $\mathrm{pp}$ and $\mathrm{p}-\mathrm{Pb}$ interactions up to the highest-multiplicity environment of the central $\mathrm{Pb}-\mathrm{Pb}$ collisions. Tracking and vertexing are performed using the Inner Tracking System (ITS), consisting of six layers of silicon detectors, and the Time Projection Chamber (TPC). The two innermost layers of the ITS and the VZERO detector (scintillation hodoscopes placed on either side of the interaction region) are used for triggering. The VZERO also provides the centrality (multiplicity) class definition in $\mathrm{Pb}-\mathrm{Pb}(\mathrm{p}-\mathrm{Pb})$ collisions, while ITS and TPC provide particle identification in the low and intermediate $p_{\mathrm{T}}$ region, respectively. Particle identification in the intermediate $p_{\mathrm{T}}$ region is provided by a Time-Of-Flight (TOF) detector. A complete description of the ALICE sub-detectors can be found in [4, 5]. In the next section the main ALICE results on the nuclear modification factors $R_{\mathrm{AA}}$ for identified strange and light-flavour hadrons are presented, together with the corresponding measurements for $\mathrm{p}-\mathrm{Pb}$ collisions.

\section{Results and discussion}

In order to study the modification of the transverse momentum spectra and to disentagle suppression due to QGP in hot nuclear matter from initial-state nuclear matter effects (e.g. Cronin enhancement [6]), the nuclear modification factor is introduced for both $\mathrm{Pb}-\mathrm{Pb}$ and $\mathrm{p}-\mathrm{Pb}$ collision systems according to the following definition:

$$
R_{\mathrm{AA}}=\frac{\mathrm{d}^{2} N_{\mathrm{AA}} / \mathrm{d} y \mathrm{~d} p_{\mathrm{T}}}{\left\langle T_{\mathrm{AA}}\right\rangle \mathrm{d}^{2} \sigma_{\mathrm{pp}}^{\mathrm{INEL}} / \mathrm{d} y \mathrm{~d} p_{\mathrm{T}}} \quad R_{\mathrm{pPb}}=\frac{\mathrm{d}^{2} N_{\mathrm{pPb}} / \mathrm{d} y \mathrm{~d} p_{\mathrm{T}}}{\left\langle T_{\mathrm{pPb}}\right\rangle \mathrm{d}^{2} \sigma_{\mathrm{pp}}^{\mathrm{INEL}} / \mathrm{d} y \mathrm{~d} p_{\mathrm{T}}}
$$

where $N_{\mathrm{AA}}\left(N_{\mathrm{pPb}}\right)$ and $\sigma_{\mathrm{pp}}^{\mathrm{INEL}}$ represent the particle yields in $\mathrm{Pb}-\mathrm{Pb}(\mathrm{p}-\mathrm{Pb})$ and the inelastic cross section in pp collisions, respectively. Both in $\mathrm{Pb}-\mathrm{Pb}$ and $\mathrm{p}-\mathrm{Pb}$ the nuclear overlap function (corresponding to $\left\langle T_{\mathrm{AA}}\right\rangle$ and $\left\langle T_{\mathrm{pPb}}\right\rangle$, respectively) is determined from the Glauber model [7] and proportional to the average number of binary nucleon-nucleon collisions $\left\langle N_{\text {coll }}\right\rangle$. By construction, $R_{\mathrm{AA}}$ and $R_{\mathrm{pPb}}$ are expected to be equal to unity in absence of nuclear effects. 
Fig. 1 shows the $R_{\mathrm{AA}}$ for $\pi, \mathrm{K}$ and $\mathrm{p}$ as a function of $p_{\mathrm{T}}$, compared with that for unidentified charged particles in six centrality classes of $\mathrm{Pb}-\mathrm{Pb}$ collisions at $\sqrt{s_{\mathrm{NN}}}=2.76 \mathrm{TeV}$ [7]. Results indicate that all particle species are equally suppressed for $p_{\mathrm{T}}$ above $10 \mathrm{GeV} / c$, hence particle ratios are similar to those from jet fragmentation in the vacuum. At lower (intermediate) $p_{\mathrm{T}}$ protons are less suppressed for all centralities. The mass dependence of the nuclear modification of the spectra is qualitatively consistent with the expectations from the presence of radial flow.

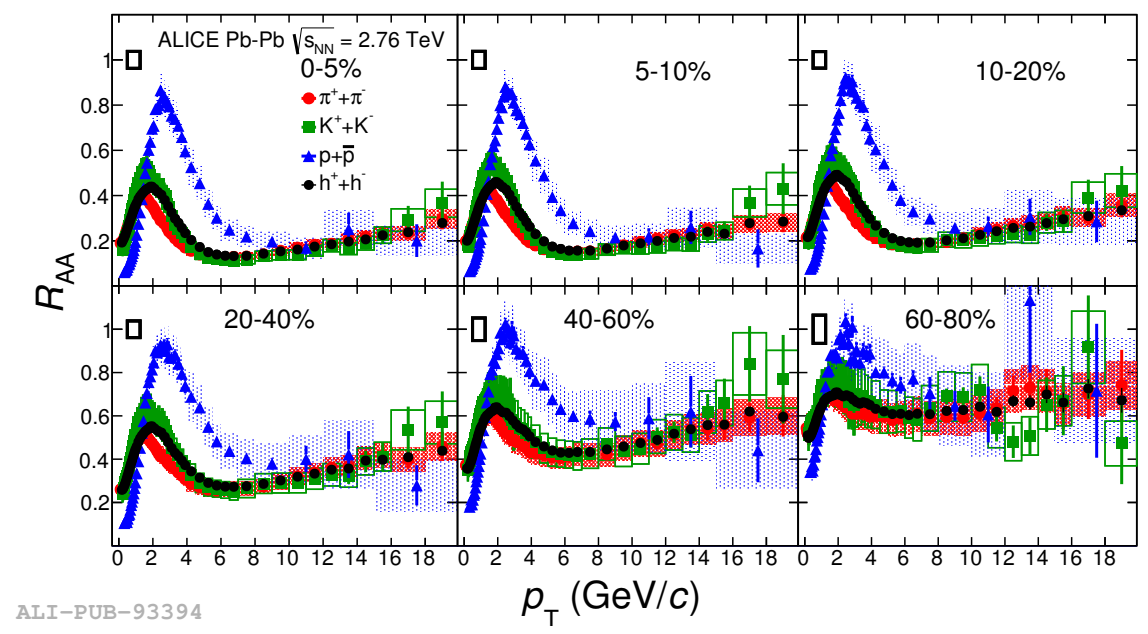

Figure 1: Nuclear modification factor $R_{\mathrm{AA}}$ for $\pi, \mathrm{K}, \mathrm{p}$ and charged particles, for different centralities in $\mathrm{Pb}-\mathrm{Pb}$ collisions at $\sqrt{s_{\mathrm{NN}}}=2.76 \mathrm{TeV}$.

The comparison of non-strange and strange baryon and meson suppression patterns at high $p_{\mathrm{T}}$ may help to disentagle the different energy loss of quarks and gluons, which arises from their different colour-coupling factors. In Fig. 2 the $R_{\mathrm{AA}}$ for $\pi, \mathrm{K}$ and $\mathrm{p}$ are compared with those for multi-strange baryons, both in central (0-10\%) and peripheral (60-80\%) $\mathrm{Pb}-\mathrm{Pb}$ collisions.
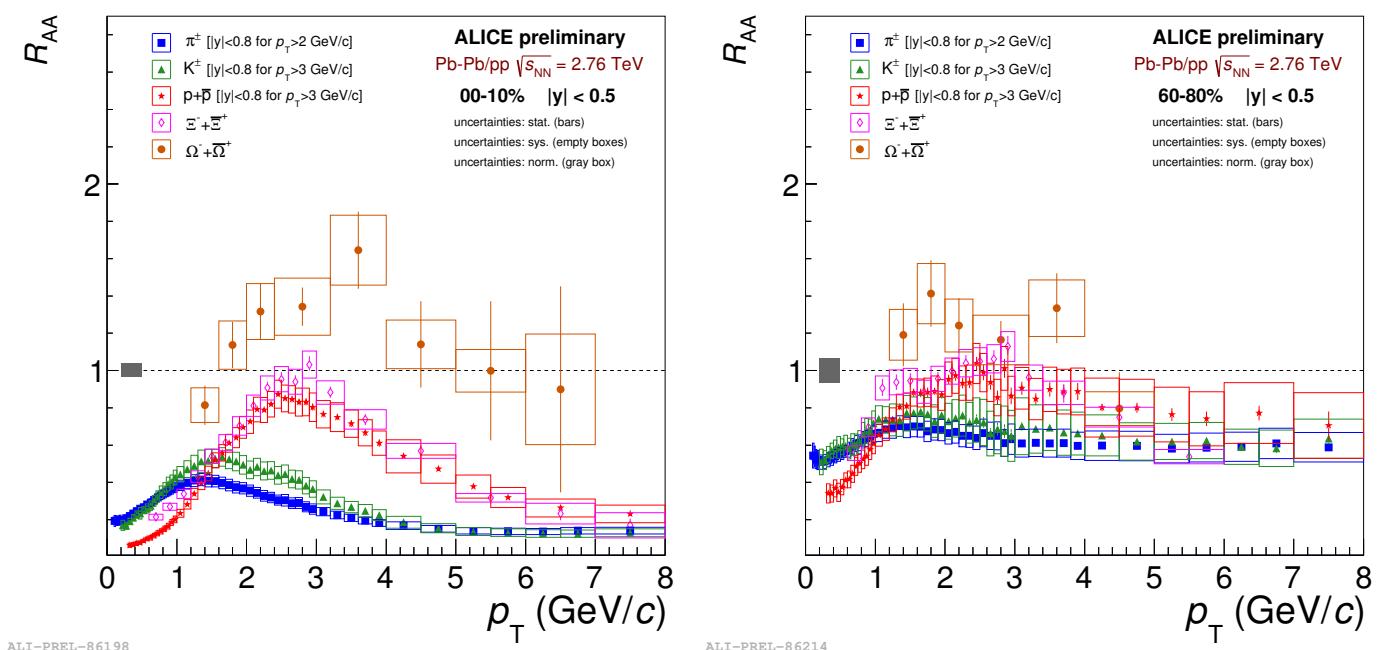

Figure 2: $R_{\mathrm{AA}}$ for $\Xi$ and $\Omega$ compared to those for charged identified particles, for 0-10\% (left) and $60-80 \%$ (right) centrality $\mathrm{Pb}-\mathrm{Pb}$ collisions at $\sqrt{s_{\mathrm{NN}}}=2.76 \mathrm{TeV}$. 
For central collisions, similarities of the curves for two groups of particles (baryons and mesons) and mass ordering at intermediate $p_{\mathrm{T}}$ can be identified. In particular, the behaviour of the nuclear modification for the $\Xi$ as a function of $p_{\mathrm{T}}$ is similar to that for protons. The large suppression at high $p_{\mathrm{T}}$, already mentioned for $\pi, \mathrm{K}$ and p, is found also for $\Xi$ up to $8 \mathrm{GeV} / c$ and with the same value common to the lighter particles. The larger $R_{\mathrm{AA}}$ values for the $\Omega$ can be related to the suppressed strangeness production in pp collisions. As expected, for peripheral events $R_{\mathrm{AA}}$ values become closer to unity and reduced differences among the different species are observed.

As already mentioned, similar measurements of nuclear modification factors in $\mathrm{p}-\mathrm{Pb}$ collisions, where the formation of a QGP state is not expected, can be used to investigate possible contributions due to (ground state) nuclear matter effects. Fig. 3 shows the $R_{\mathrm{pPb}}$ for charged $\pi, \mathrm{K}$ and $\mathrm{p}$ up to $15 \mathrm{GeV} / c$ and for $\Xi$ and $\Omega$ up to 7 and $5 \mathrm{GeV} / c$, respectively, in non-single diffractive (NSD) $\mathrm{p}-\mathrm{Pb}$ collisions.

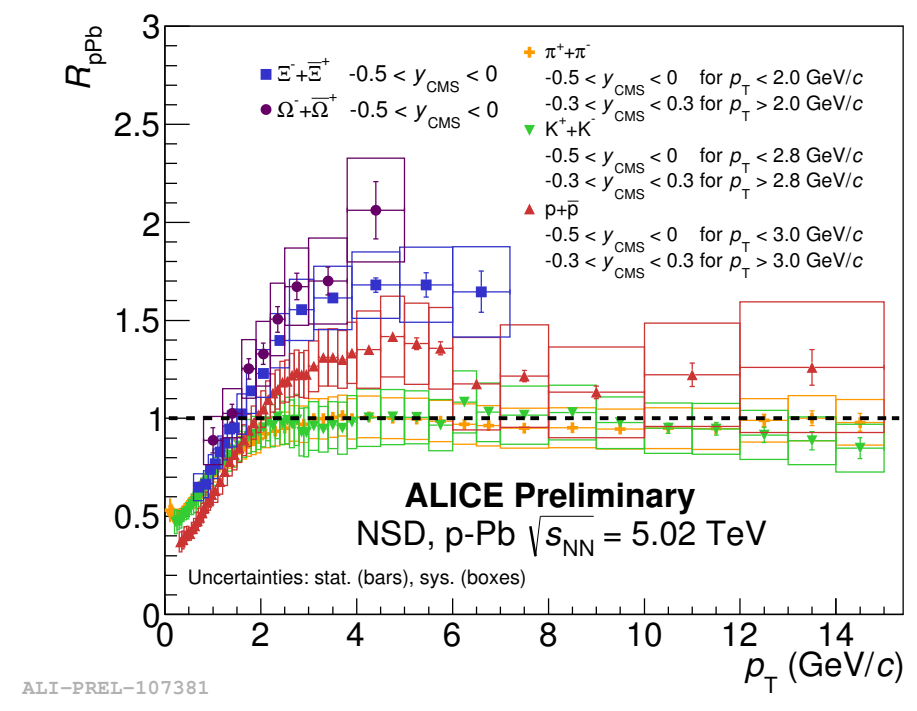

Figure 3: $R_{\mathrm{pPb}}$ for $\pi, \mathrm{K}, \mathrm{p}$ and multi-strange baryons in NSD p-Pb collisions at $\sqrt{s_{\mathrm{NN}}}=5.02 \mathrm{TeV}$.

The nuclear modification factors for pions and kaons are flat over the full measured $p_{\mathrm{T}}$ range above $3 \mathrm{GeV} / c$ and do not differ from that of unidentified charged particles within the systematic uncertainties. At intermediate $p_{\mathrm{T}}$ (in the Cronin region), a mass ordering is observed, with higher mass particles exibiting a larger $R_{\mathrm{pPb}}$. In $\mathrm{Pb}-\mathrm{Pb}$, this effect is interpreted as due to radial flow. Only p, $\Xi$ and $\Omega$ exibit a Cronin peak, which indicates that the enhancement seen for charged particles is mostly driven by protons. For larger transverse momenta $\left(p_{\mathrm{T}}>8 \mathrm{GeV} / c\right)$ all particles exibit an $R_{\mathrm{pPb}}$ consistent with unity, indicating that the suppression in $\mathrm{Pb}-\mathrm{Pb}$ is a hot matter effect.

\section{Conclusions}

The ALICE Collaboration has measured the nuclear modification factors in both $\mathrm{Pb}-\mathrm{Pb}$ and $\mathrm{p}-\mathrm{Pb}$ collisions at the LHC. The $R_{\mathrm{AA}}$ of charged particles shows suppression for all centralities, with the strongest effect for the most central collisions, decreasing towards higher $p_{\mathrm{T}}$ with the expected reduction of the relative parton energy loss. No particle species dependence is observed 
at high $p_{\mathrm{T}}$. At lower $p_{\mathrm{T}}$, protons are less suppressed and the mass ordering reflects radial flow. Similar behaviour to protons is found for the $\Xi$, while for the $\Omega$ a larger $R_{\mathrm{AA}}$ may indicate a greater effect of strangeness suppression in pp collisions. The nuclear modification in $\mathrm{p}-\mathrm{Pb}$ collisions shows a moderate Cronin enhancement for protons and multi-strange baryons at intermediate $p_{\mathrm{T}}$. At high $p_{\mathrm{T}}$ the $R_{\mathrm{pPb}}$ is consistent with binary collision scaling, indicating that the strong suppression observed in $\mathrm{Pb}-\mathrm{Pb}$ is due to final state effects.

\section{References}

[1] B. Müller et al., Ann. Rev. Nucl. Part. Sci. 62, 361 (2012).

[2] M. Gyulassy et al., Nucl. Phys. Letters B 571, 197 (2000).

[3] P. Levai et al., Nucl. Phys. A 698, 631 (2002).

[4] K. Aamodt et al. (ALICE Collaboration), JINST 3, S08002 (2008).

[5] B. Abelev et al. (ALICE Collaboration), Int. J. Mod. Phys. A 291430044 (2014).

[6] B. Abelev et al. (ALICE Collaboration), Phys. Rev. Lett. 110082302 (2013).

[7] M. Miller et al., Ann. Rev. Nucl. Part. S. 57, 205 (2007).

[8] J. Adam et al. (ALICE Collaboration), Phys. Rev. C 93, 034913 (2015). 